\title{
Non-Parametric Classifiers Based Emotion Classification Using Electrodermal Activity and Modified Hjorth Features
}

\author{
Yedukondala Rao VEERANKI ${ }^{\mathrm{a}, 1}$, Nagarajan GANAPATHY ${ }^{\mathrm{b}}$, and Ramakrishnan \\ SWAMINATHAN ${ }^{\mathrm{a}}$ \\ a Biomedical Engineering Group, Department of Applied Mechanics, \\ Indian Institute of Technology Madras, Chennai, Tamil Nadu, India \\ ${ }^{b}$ Peter L. Reichertz Institute for Medical Informatics of TU Braunschweig and \\ Hannover Medical School, Germany
}

\begin{abstract}
In this work, an attempt has been made to classify various emotional states in Electrodermal Activity (EDA) signals using modified Hjorth features and non-parametric classifiers. For this, the EDA signals are collected from a publicly available online database. The EDA is decomposed into SCL (Skin Conductance Level) and SCR (Skin Conductance Response). Five features, namely activity, mobility, complexity, chaos, and hazard, collectively known as modified Hjorth features, are extracted from SCR and SCL. Four non-parametric classifiers, namely, random forest, k-nearest neighbor, support vector machine, and rotation forest, are used for the classification. The results demonstrate that the proposed approach can classify the emotional states in EDA. Most of the features exhibit statistical significance in discriminating emotional states. It is found that the combination of modified Hjorth features and rotation forest is most accurate in classifying the emotional states. Thus, the result demonstrates that this method can recognize valence and arousal dimensions under various clinical conditions.
\end{abstract}

Keywords. Electrodermal activity, Emotional states, Modified Hjorth features, Non-parametric classifiers

\section{Introduction}

Emotion is a complex behavioral phenomenon that influences thought and behavior [1]. According to World Health Organization, the emotional abnormality, namely major depressive disorder is predicted to become the leading cause of disability by 2030 for around 20 percent of the population over the course of life [2]. Automated analysis and recognition of emotions can help understand various neurodevelopment disorders such as major depressive disorder and autism. Emotions are described using two popular dimensions, namely valence and arousal. The valence expresses the amount of negative and positive felt by the subject, and arousal characterizes the emotion intensity [3].

Emotions are quantified from various vital parameters such as Electrodermal activity (EDA), Blood Pressure (BP), breathing, Electroencephalograms (EEG),

\footnotetext{
${ }^{1}$ Corresponding Author: Yedukondala Rao Veeranki; Biomedical Engineering Group, Department of Applied Mechanics, Indian Institute of Technology Madras, Chennai, India, E-mail: ykraoveeranki@gmail.com.
} 
Electromyograms (EMG), and Electro-cardiograms (ECG). These methods have attracted more attention since the measurements can be quantified, and they cannot be easily controlled. Among the vital signals, EDA measurements are simple and convenient to use as wearable devices [4].

EDA refers to variation in electrical properties of skin, which is strongly correlated with eccrine sweat gland activity of the autonomic nervous system. Unlike other physiological signals, EDA signals are free from parasympathetic aspects of the autonomic nervous system. However, the dynamics and morphological characteristics of EDA signals are complex and exhibit inter-subject and intra-subject variability. EDA signal primarily contains two components, namely tonic and phasic. Tonic component, slow variation of skin conductance, or Skin Conductance Level (SCL) and phasic component, relatively fast variation of skin conductance, or Skin Conductance Responses (SCR). The algorithm, called the convex optimization approach (cvxEDA), gives better results for decomposing EDA at different noise levels [5].

This study explores the feasibility of modified Hjorth features to recognize the valence and arousal dimensions in EDA signals.

\section{Materials and methods}

The proposed methodology for analyzing EDA signal using modified Hjorth parameters is pictorially represented in Figure 1.

\subsection{Database}

The EDA signals are collected from online public DEAP database for the analysis. The database it contains 48 channels of multiple physiological signals obtained from 32 subjects, each 48-minute length, and sampled at $128 \mathrm{~Hz}$. The signals are acquired from the subjects while watching 40 different audio-visual stimuli of one-minute length under the well-defined protocol [6].

\subsection{Feature extraction}

The EDA signals are decomposed into SCR and SCL using cvxEDA algorithm and the modified Hjorth descriptors, namely activity, mobility, complexity, chaos, and hazard, are extracted. The mathematical expressions are given below [7],[8]:

Activity (AC): It is a measure of the average of the squared differences from the amplitude of the signal [7]. It can be mathematically expressed as:

$$
\mathrm{AC}=\sum_{\mathrm{n}=1}^{\mathrm{N}}(\mathrm{x}(\mathrm{n})-\mu)^{2} / \mathrm{N}
$$

Where $N$ represents thelength of signal $x(n)$ and $\mu$ denotes the mean of $x(n)$

Mobility (MO): is the ratio of the variance of the first derivative of the signal and the signal [7]. The mobility is given as:

$$
\mathrm{MO}=\sqrt{\mathrm{AC}\left(\mathrm{x}^{\prime}(\mathrm{n})\right) / \mathrm{AC}(\mathrm{x}(\mathrm{n}))}
$$

Where $x^{\prime}(n)$ represents first-order differentiation of signal $x(n)$.

Complexity (CO): It measures the change in the frequency of the signal and indicates how the shape of the signal is similar to pure sinewave [7]. It is given by: 


$$
\mathrm{CO}=\mathrm{MO}\left(\mathrm{x}^{\prime}(\mathrm{n})\right) / \mathrm{MO}(\mathrm{x}(\mathrm{n}))
$$

Chaos (CH): It is given as the ratio between the $\mathrm{CO}$ of the time series derivative and the $\mathrm{CO}$ of the series itself [8]. It is expressed as:

$$
\mathrm{CH}=\mathrm{CO}\left(\mathrm{x}^{\prime}(\mathrm{n})\right) / \mathrm{CO}(\mathrm{x}(\mathrm{n}))
$$

Hazard (HZ): It is given as the ratio between the $\mathrm{CH}$ of the time series derivative and the $\mathrm{CH}$ of the series itself [8]. It can be mathematically expressed as:

$$
\mathrm{HZ}=\mathrm{CH}\left(\mathrm{x}^{\prime}(\mathrm{n})\right) / \mathrm{CH}(\mathrm{x}(\mathrm{n}))
$$

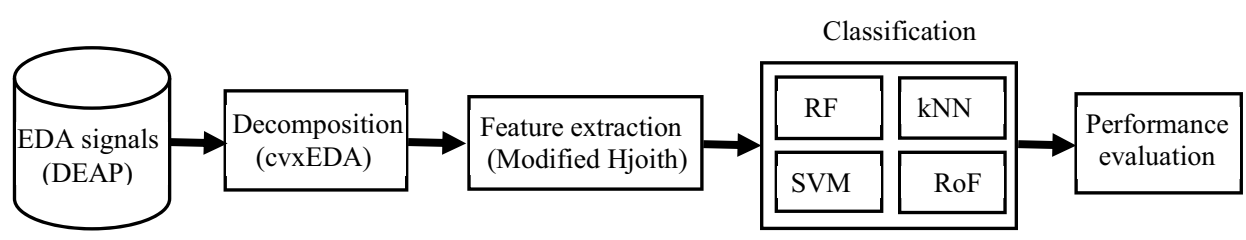

Figure 1. Block diagram of the proposed methodology

Further, statistical analysis has been performed to know the significance of features for differentiating arousal and valence dimensions

\subsection{Classification}

The extracted features are fed to the four non-parametric classifiers, namely k-Nearest Neighbor (kNN), Support Vector Machine (SVM), Random Forest (RF), and Rotation Forest (RoF) classifiers. The kNN determines k objects nearest to the test object in the training data and assigns a class based on the neighbors. To distinguish between two classes, SVM uses a discriminating hyperplane. RF takes averages of the multiple decision tree predictions for making decisions. RoF divides the given set of features into various subsets to extract principal components. The new features for a decision tree are generated by carrying out the same rotations. The performance metrics, namely Accuracy (Acc), Precision (Prec), Recall (Rec), and F-measure (F-m) are evaluated from the classifiers [9].

\section{Results and Discussion}

The representative EDA signals for the dominant arousal, neutral arousal, positive valence, and negative valence classes are shown in Figure 2. It is observed that across the subjects, the amplitude and frequency of the signals are different. These differences are dependent on the subject and are attributed to multiple parameters, such as per-gland sweat, density, distribution, and size of sweat gland. Large fluctuations are seen in some subjects. However, it is not observed in all the cases. This may be due to anthropometric variations and distribution of sweat glands. Increasing trend in amplitude is observed in Figure 2 (b). It might be attributed to continuous activation of sweat glands due to emotional stimuli. Higher frequency content is observed in Figure 2 (c).

The representative SCR component for the dominant arousal, neutral, positive, and negative valence classes are shown in Figure 3. It is observed that the subtle variations in conductance are higher in positive valence and arousal. It may be attributed due to 
high sweat gland activity triggered by postganglionic sudomotor fibers. It is also seen that the conductance is low for neutral and negative valence. For dominant arousal and positive valence, a positive rise in amplitude is observed along with fluctuations, whereas minimal and limited fluctuations are observed in the neutral and negative valence.

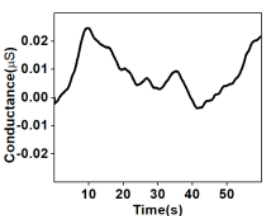

(a)

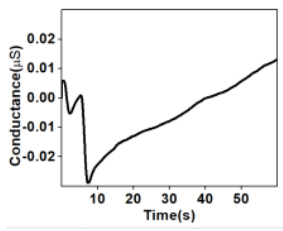

(b)

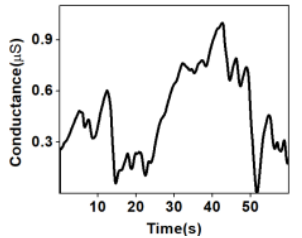

(c)

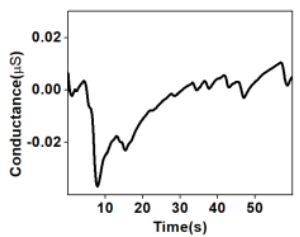

(d)

Figure 2. The Representative EDA signal of a subject in (a) dominant arousal, (b) neutral arousal, (c) positive valence, and (d) negative valence emotional states

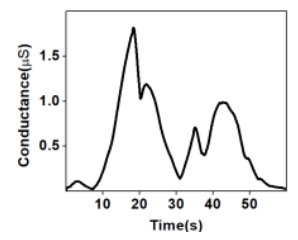

(a)

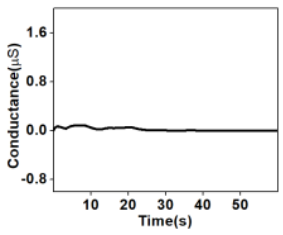

(b)

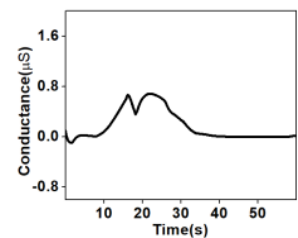

(c)

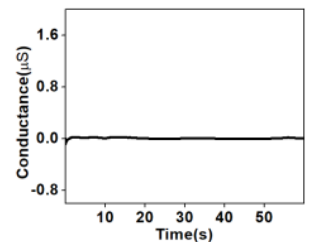

(d)

Figure 3. The Representative SCR signal of a subject in (a) dominant arousal, (b) neutral arousal, (c) positive valence, and (d) negative valence emotional states

The representative SCL component for the dominant arousal, neutral arousal, positive valence, and negative valence classes are shown in Figure 4. Few fluctuations are observed in positive valence and arousal when compared to neutral and negative valence. It is also observed that the SCR conductance varies faster than the SCL, and the conductance is relatively higher for the SCR than the SCL.

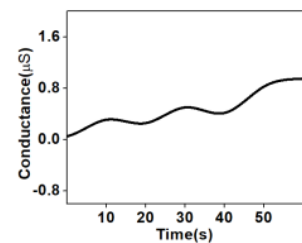

(a)

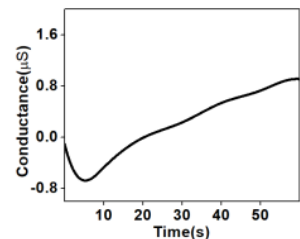

(b)

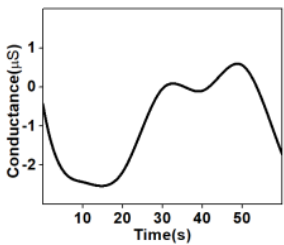

(c)

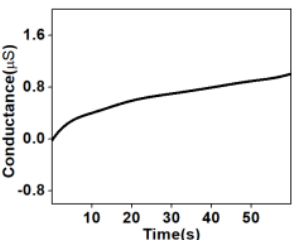

(d)

Figure 4. The representative SCL signal of a subject in (a) dominant arousal, (b) neutral arousal, (c) positive valence, and (d) negative valence emotional states

The statistical significance of modified Hjorth parameters for valence and arousal dimensions of SCR and SCL are calculated using the Wilcoxon rank-sum test. It is observed that the features, namely activity, and mobility are significant $(p<0.05)$ in discriminating the valence dimension using SCR. The features, namely activity, mobility, complexity, chaos, and hazard, are significant $(\mathrm{p}<0.05)$ in differentiating the valence dimension using SCL.

The extracted modified Hjorth parameters are fed to four non-parametric classifiers, namely, RF, kNN, SVM, and RoF. The classification efficiency of the computed features is shown in Table 2 and Table 3. It is found that the RoF yields the highest accuracy of $63.28 \%$ and $53.82 \%$ for classifying arousal and valence dimension respectively, in SCL. Table 3 represents the classification performance of SCR. RoF yields the highest accuracy of $63.04 \%$ and $53.90 \%$ for classifying arousal and valence dimensions. 
Table 1. The performance classification [\%] of SCL features with various non-parametric classifiers

\begin{tabular}{ccccccccc}
\hline \multirow{2}{*}{$\begin{array}{c}\text { Classif } \\
\text { ier }\end{array}$} & \multicolumn{9}{c}{ SCL } \\
\cline { 2 - 9 } & Acc & Prec & Rec & F-m & Acc & Prec & Rec & F-m \\
\hline RF & 49.76 & 49.30 & 49.80 & 49.54 & 55.00 & 51.30 & 55.00 & 53.08 \\
kNN & 46.40 & 46.40 & 46.40 & 46.40 & 46.90 & 47.40 & 47.00 & 47.19 \\
SVM & 51.40 & 49.50 & 51.40 & 50.43 & 61.79 & 50.90 & 61.80 & 55.82 \\
RoF & $\mathbf{5 3 . 8 2}$ & $\mathbf{5 2 . 3 0}$ & $\mathbf{5 3 . 8 0}$ & $\mathbf{5 3 . 0 3}$ & $\mathbf{6 3 . 2 8}$ & $\mathbf{7 6 . 8 0}$ & $\mathbf{6 3 . 3 0}$ & $\mathbf{6 9 . 3 9}$ \\
\hline
\end{tabular}

Table 2. The performance classification [\%] of SCR features with various non-parametric classifiers

\begin{tabular}{|c|c|c|c|c|c|c|c|c|}
\hline \multirow{3}{*}{$\begin{array}{c}\text { Classif } \\
\text { ier }\end{array}$} & \multicolumn{8}{|c|}{ SCR } \\
\hline & \multicolumn{4}{|c|}{ Valence } & \multicolumn{4}{|c|}{ Arousal } \\
\hline & Acc & Prec & Rec & F-m & Acc & Prec & Rec & F-m \\
\hline RF & 49.76 & 49.40 & 49.80 & 49.59 & 60.15 & 57.00 & 60.20 & 58.55 \\
\hline kNN & 51.48 & 51.40 & 51.50 & 51.44 & 56.25 & 56.30 & 56.30 & 56.30 \\
\hline SVM & 53.43 & 48.50 & 53.40 & 50.83 & 63.04 & 39.80 & 63.00 & 48.78 \\
\hline RoF & 53.90 & 59.80 & 53.90 & 56.69 & 63.04 & 39.80 & 63.00 & 48.78 \\
\hline
\end{tabular}

\section{Conclusion}

This work aims to analyze modified Hjorth features performance to classify valence and arousal dimensions in EDA and compare its performance using non-parametric classifiers. The results demonstrate that the Hjorth features extracted in the time-domain can classify the arousal and valence dimensions of EDA. The features obtained are statistically significant in differentiating valence dimension. The combination of modified Hjorth features and RoF is most accurate in classifying the arousal and valence dimensions. The methodology adopted in this work could well be extended to larger data samples.

\section{References}

[1] Boucsein W. Electrodermal activity. Springer Science \& Business Media; 2012 Feb 2.

[2] World Health Organization. Depression and other common mental disorders: global health estimates. World Health Organization; 2017.

[3] Eerola T, Vuoskoski JK. A comparison of the discrete and dimensional models of emotion in music. Psychology of Music. 2011 Jan;39(1):18-49.

[4] Ganapathy N, Veeranki YR, Swaminathan R. Convolutional Neural Network based Emotion Classification using Electrodermal Activity Signals and Time-Frequency Features. Expert Systems with Applications. 2020 May 30;113571.

[5] Posada-Quintero HF, Chon KH. Innovations in electrodermal activity data collection and signal processing: A systematic review. Sensors. 2020 Jan;20(2):479.

[6] Koelstra S, Muhl C, Soleymani M, Lee JS, Yazdani A, Ebrahimi T, Pun T, Nijholt A, Patras I. Deap: A database for emotion analysis; using physiological signals. IEEE transactions on affective computing. 2011 Jun 9;3(1):18-31.

[7] Leite JP, Moreno RL. Heartbeat classification with low computational cost using Hjorth parameters. IET Signal Processing. 2017 Nov 21;12(4):431-8.

[8] Wannawijit I, Kaiwansil S, Ruthaisujaritkul S, Yingthawornsuk T. ECG Classification with Modification of Higher-Order Hjorth Descriptors. In2019 15th International Conference on Signal-Image Technology \& Internet-Based Systems (SITIS) 2019 Nov 26; p. 564-571.

[9] Karthick PA, Ghosh DM, Ramakrishnan S. Surface electromyography based muscle fatigue detection using high-resolution time-frequency methods and machine learning algorithms. Computer methods and programs in biomedicine. 2018 Feb 1;154:45-56. 